\title{
TCF3, a novel positive regulator of osteogenesis, plays a crucial role in miR-17 modulating the diverse effect of canonical Wnt signaling in different microenvironments
}

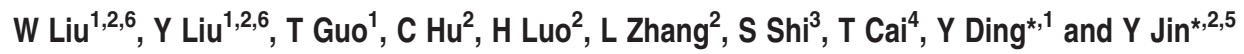

Wnt signaling pathways are a highly conserved pathway, which plays an important role from the embryonic development to bone formation. The effect of Wnt pathway on osteogenesis relies on their cellular environment and the expression of target genes. However, the molecular mechanism of that remains unclear. On the basis of the preliminary results, we observed the contrary effect of canonical Wnt signaling on osteogenic differentiation of periodontal ligament stem cells (PDLSCs) in the different culture environment. Furthermore, we found that the expression level of miR-17 was also varied with the change in the culture environment. Therefore, we hypothesized that miR-17 and canonical Wnt signaling may have potential interactions, particularly the inner regulation relationship in different microenvironments. In this paper, we observed that canonical Wnt signaling promoted osteogenesis of PDLSCs in the fully culture medium, while inhibited it in the osteogenic differentiation medium. Interestingly, alteration in the expression level of endogenous miR-17 could partially reverse the different effect of canonical Wnt signaling. Furthermore, the role of miR-17 was because of its target gene TCF3 (transcription factor 3), a key transcription factor of canonical Wnt pathway. Overexpression of TCF3 attenuated the effect of miR-17 on modulating canonical Wnt signaling. Finally, we elucidated that TCF3 enhanced osteogenesis both in vitro and in vivo. In brief, the different level of miR-17 was the main cause of the different effect of canonical Wnt signaling, and TCF3 was the crucial node of miR-17-canonial Wnt signaling regulation loop. This understanding of microRNAs regulating signaling pathways in different microenvironments may pave the way for fine-tuning the process of osteogenesis in bone-related disorders.

Cell Death and Disease (2013) 4, e539; doi:10.1038/cddis.2013.65; published online 14 March 2013

Subject Category: Experimental medicine

During development of the skeleton and formation of bone tissue, a handful of pathways precisely govern stem cell differentiation and osteogenesis, and their perturbations account disproportionally for bone-related diseases. ${ }^{1,2}$ Among the several pathways, the effect of Wnt signaling was the most controversial. ${ }^{3,4}$ It has been reported that canonical Wnt pathway promotes the osteogenesis of murine MSCs and osteoprogenitor cells through the upregulation of osteoblast-related genes. ${ }^{5,6}$ On the other side, de Boer et al. ${ }^{7}$ showed that activation of canonical Wnt signaling suppressed dexamethasone-induced osteogenesis in hMSCs. Interestingly, low-dose Wnt3a resulted in a significant relative increase in osteogenic differentiation. Nevertheless, high-dose Wnt3a partially inhibited osteogenic differentiation. ${ }^{8}$ This dual effect of canonical Wnt signaling suggested that the intensity of Wnt signals can lead to different or even opposite biological functions. Furthermore, we demonstrated that canonical Wnt signaling promoted or attenuated osteogenic differentiation of periodontal ligament stem cells (PDLSCs) depending on the culture environment described previously. ${ }^{9}$ These controversial findings were similar to the Ling et al.'s research, ${ }^{10}$ suggesting that the effect of canonical Wnt signaling on MSCs' osteogenic differentiation may be affected by the microenvironment. However, the molecular mechanism and cellular target of this is not yet fully understood.

\footnotetext{
${ }^{1}$ Department of Orthodontics, School of Stomatology, The Fourth Military Medical University, Xi'an, Shaanxi, People's Republic of China; ${ }^{2}$ Research and Development Center for Tissue Engineering, The Fourth Military Medical University, Xi'an, Shaanxi, People's Republic of China; ${ }^{3}$ Center for Craniofacial Molecular Biology, University of Southern California School of Dentistry, 2250 Alcazar Street, CSA 103, Los Angeles, CA, USA; ${ }^{4}$ Experimental Medicine Section, Oral Infection and Immunity Branch, National Institute of Dental and Craniofacial Research (NIDCR), National Institutes of Health, Bethesda, MD, USA and ${ }^{5}$ Department of Oral Histology and Pathology, School of Stomatology, The Fourth Military Medical University, Xi'an, Shaanxi, People's Republic of China

${ }^{*}$ Corresponding author: Y Jin, 145 West Changle Road, Xi'an, Shaanxi 710032, People's Republic of China. Tel: + 862984776147 ; Fax: + 86 29 83218039;

E-mail: yanjin @fmmu.edu.cn

Or Y Ding, 145 West Changle Road, Xi'an, Shaanxi 710032, People's Republic of China. Tel: + 8629 84776131; Fax: + 862984776131 ;

E-mail: Dingyin@fmmu.edu.cn

${ }^{6}$ These authors contributed equally to this work

Keywords: transcription factor 3; miR-17; canonical Wnt signaling; osteogenesis; microenvironment

Abbreviations: hPDLSCs, human periodontal ligament mesenchymal stem cells; miRNA/miR, microRNA; mRNA, messenger RNA; $3^{\prime}$-UTR, $3^{\prime}$-untranslated region; PCR, polymerase chain reaction; WT, wild type; MUT, mutated type; Runx2, runt-related transcription factor 2; ALP, alkaline phosphatase; TCF3, transcription factor 3; TCF4, transcription factor 4; LEF1, lymphoid enhancer-binding factor 1; sh-TCF3, lentiviral construct for downregulation of TCF3; plenti-TCF3, lentiviral construct for upregulation of TCF3

Received 11.12.12; revised 20.1.13; accepted 24.1.13; Edited by M Agostini
} 
On activation of the canonical Wnt pathway, $\beta$-catenin binds to the TCF/LEF (T-cell factor/lymphoid enhancer-binding factor) transcription factors and mediates the downstream target genes' expression. ${ }^{11,12}$ Among the four members, TCF1 and TCF4 were proved to activate runt-related transcription factor 2 (Runx2) expression directly in MSCs for the control of osteoblast differentiation and skeletal development. ${ }^{13,14}$ In contrast, LEF1 repressed the expression level of Runx2 and acted as a negative regulator of osteogenic differentiation. ${ }^{15}$ TCF3 is considered to be predominantly a transcriptional repressor in complex with $\beta$-catenin, but the role of it in modulating osteogenesis remains unclear.

Recent evidences have shown that osteogenic differentiation is also regulated by post-transcriptional mechanisms, most significantly by microRNAs (miRNAs). ${ }^{16-18}$ Stem cells that develop into osteoblasts have already been shown to be controlled by various miRNAs. For instance, miR-204/211 targets Runx2 and inhibits osteoblastic differentiation of bonemarrow-derived MSCs. ${ }^{19}$ miR-335 also negatively regulates Runx2 to inhibit the osteoblastic differentiation of hMSCs isolated from the bone marrow, adipose tissue and articular cartilage. ${ }^{20}$ Moreover, miR-20a can promote the osteogenesis of hMSCs via BMP signaling. ${ }^{21}$ Previously, we demonstrated that miR-17 acted as a negative regulator of osteogenesis in the normal microenvironment. However in the inflammation microenvironment, overexpression of miR-17 could promote the osteogenic differentiation. It is suggested that miR-17 has a role in the stimulation or inhibition of osteoblast differentiation that depends on the microenvironment. ${ }^{22}$ As the miRNA processing was faster than the signaling pathway cascade activation, miRNAs were more sensitive to the microenvironment. Hence, we hypothesize that miR-17 may act as an important factor in regulating the different effect of canonical Wnt pathway on osteogenesis from the post-transcriptional level.

In this study, we testified our hypothesis and elucidated that the opposite effect of canonical Wnt signaling in the fully cultured medium and osteogenic differentiation medium was mainly caused by different expression level of miR-17. By modifying the expression level of endogenous miR-17, the function of canonical Wnt signaling was reversed. Furthermore, we identified that the key transcriptional factor of canonical Wnt pathway, TCF3, was a direct target gene of miR-17. Meanwhile, overexpression of TCF3 could partially attenuate the effect of miR-17 upregulation on reversal of the canonical Wnt signaling. Finally, TCF3 was proved for the first time to promote the osteogenic differentiation in vitro and ectopic bone formation in vivo. Our results suggest that miR-17 modulates the contrary effect of canonical Wnt signaling on osteogenesis in different microenvironments mainly through targeting TCF3 directly, which has been proven to act as a positive regulator of osteogenesis.

\section{Results}

Identification of human PDLSCs. The mesenchymal stem cells isolated from PDLSCs retained a typical fibroblastic spindle shape and were capable of forming CFU-F from single cells (Supplementary Figure S1a).
Immunocytochemical staining and flow cytometric analyses showed that the PDLSCs expressed the mesenchymal cell marker vimentin as well as the stem cell markers STRO-1, CD146, CD29, CD31, CD34, CD45, CD90 and CD105 (Supplementary Figures S1a and b).

To investigate the multiple differentiation potential of the PDLSCs, at passage three, the cells were cultured in osteogenic differentiation media for 21 days. Numerous mineralized nodules stained by alizarin red were formed by day 21 (Supplementary Figure S1c). The expression levels of osteoblast-related genes were measured, including Runx2, which is a critical transcription factor for osteogenic differentiation because it provides a permanent directional signal for lineage determination; alkaline phosphatase (ALP) is an important index for osteogenic differentiation that is not controlled by Runx2/Cbfa1, osteocalcin and osteopontin, mature osteoblast markers. The expression of these marker genes was significantly increased after 21 days of induction (Supplementary Figure S1d). After adipogenic induction, the amount of lipid droplets gathered in the cytoplasm and the expression of the specific adipogenic markers, peroxisome proliferator-activated receptor- $\gamma$ and lipoprotein lipase (LPL), showed an obvious upregulation (Supplementary Figure S1d) at day 21 .

Canonical Wnt signaling has different effect on osteogenesis depending on the culture environment. As the in vitro effects of canonical Wnt signaling on osteogenic differentiation of mesenchymal stem cells are dose-dependent, ${ }^{8}$ these may come from the difference in culture conditions. ${ }^{10}$ We used $25 \mathrm{l}$ or $50 \mathrm{ng} / \mathrm{ml}$ recombinant human Wnt3a to activate the canonical Wnt pathway. The results showed that in fully culture medium, Wnt3a could functionally affect the expression of $\beta$-catenin in the nucleus of PDLSCs and there was no statistical difference between the two doses. However, when PDLSCs were cultured in the osteogenic differentiation medium, the expression level of $\beta$ catenin in the nucleus was significantly decreased in the Wnt3a $50 \mathrm{ng} / \mathrm{ml}$ group compared with the Wnt3a $25 \mathrm{ng} / \mathrm{ml}$ group (Supplementary Figure S2). These results were consistent with our previous research. ${ }^{9}$ Therefore, the Wnt3a $50 \mathrm{ng} / \mathrm{ml}$ group has been chosen for further study.

We observed that Wnt3a promoted the ALP activity and staining in the culture medium. However, ALP activity and staining were inhibited obviously in the osteogenic differentiation medium (Figure 1a). The mRNA and protein levels of ALP and Runx2 were also consistent with this result (Figure 1b). These data suggested that canonical Wnt signaling showed the opposite effect on osteogenic differentiation of PDLSCs in different culture environment.

MiR-17 partially reverses the effect of canonical Wnt signaling on osteogenic differentiation in PDLSCs. Previously, we found that miR-17 acted as a negative regulator of PDLSCs' osteogenic differentiation (Figure 2b) in the normal microenvironment. We also proved that the microenvironment determined the role of $\mathrm{miR}-17$ in the stimulation or inhibition of osteoblast differentiation. ${ }^{22}$ Therefore, we testified the expression pattern of miR-17 after the PDLSCs were cultured in the fully culture medium or 
a
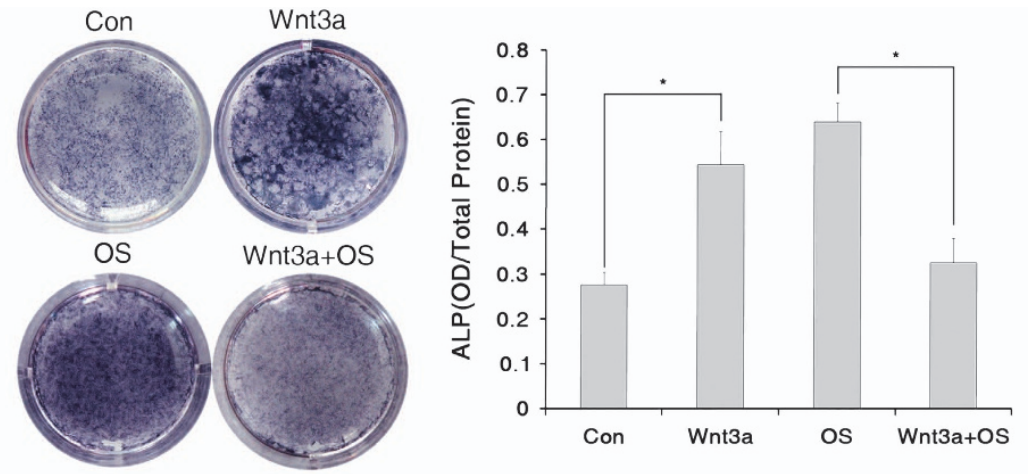

b
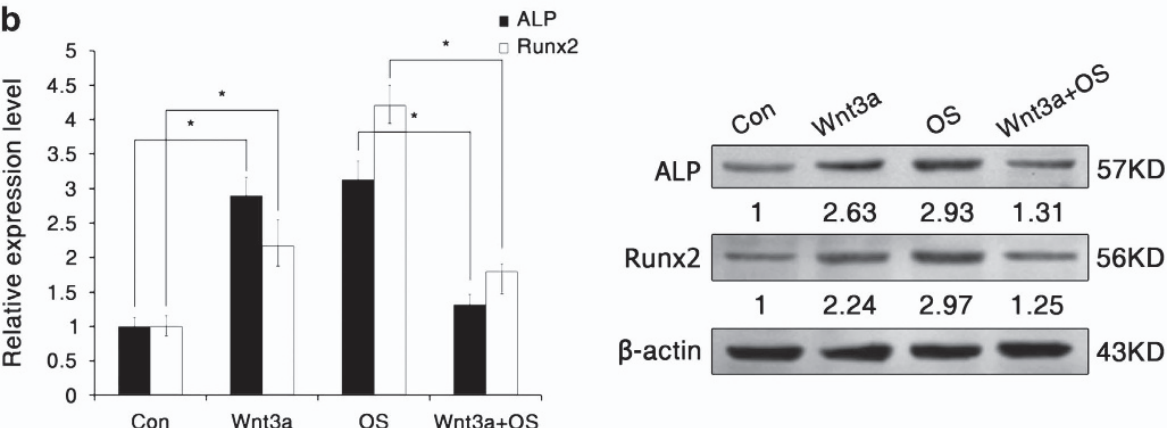

Figure 1 Canonical Wnt signaling had an opposite effect on osteogenic differentiation of PDLSCs in different culture environment. PDLSCs were cultured in the fully culture medium or osteogenic differentiation medium with $50 \mathrm{ng} / \mathrm{ml}$ recombinant Wnt3a for 7 days. (a) ALP staining and activity analyses were performed at day 7 . (b) The expression levels of ALP and Runx2 were measured at the indicated time by real-time PCR and western blot. The expression levels of mRNA and protein were normalized to $\beta$-actin. The data are shown as mean \pm S.D. ${ }^{*} P<0.05, n=3$. Con, control; OD, optical density; OS, osteogenic induction; Wnt3a, $50 \mathrm{ng} / \mathrm{ml}$ recombinant human Wnt3a

osteogenic differentiation medium with Wnt3a. After 7 days, in the fully culture medium, the level of miR-17 was decreased nearly onefold after adding Wnt3a. However, Wnt3a increased the level of miR-17 more than twofold in the osteogenic differentiation medium (Figure 2a). Thereafter, we changed the endogenous level of miR-17. ALP activity and staining results showed that Wnt3a promoted the osteogenic differentiation of PDLSCs in the culture microenvironment. These were demonstrated by the increased level of ALP and Runx2. However, overexpression of miR-17 by pre-miR-17 could attenuate this effect. The expression of ALP and Runx2 was decreased nearly 1.8- and 2-fold, respectively. However, it was still higher than the control group. Furthermore, downregulation of miR-17 via anti-miR17 could rescue the effect of Wnt3a on inhibition of osteogenic differentiation of PDLSCs in the induction microenvironment. Polymerase chain reaction (PCR) and western blot showed that the expression of ALP and Runx2 was increased 1.9- and 1.5-fold after being transfected with anti-miR-17, which was still lower than the osteogenic induction group (Figures 2c and d).

Canonical Wnt pathway-related transcription factor TCF3 is a direct target gene of miR-17. To clarify the mechanism underlying the function of miR-17, we first focused on the identification of potential targets of miR-17. Our previous research found that Smad ubiquitin regulatory factor 1 (Smurf1) was a target gene of miR-17 and acted as a negative regulator of osteogenic differentiation. ${ }^{22}$ However, the PCR and western blot analysis showed that the expression level of Smurf1 was not affected by canonical Wnt pathway (Supplementary Figure S3). Therefore, we screened the target gene prediction software, and noticed that three important transcription factors of canonical Wnt pathway (TCF3, TCF4 and LEF1) were the potential target genes of miR-17 (Table 1).

When the cellular expression levels of miR-17 level were modified by pre-miR-17 and anti-miR-17, only the TCF3 expression was observed with the exactly opposite pattern to that of the miR-17 (Figure 3a). Furthermore, the peaked expression level for TCF3 during the osteogenic differentiation was at day 7 (Figure 3d), whereas the lowest level for miR-17 was on the same time point (Figure 3e), suggesting their potential correlation. As a putative miR-17-binding site was identified in the TCF3 $3^{\prime}$-untranslated region $\left(3^{\prime}\right.$-UTR) region (Figure $3 \mathrm{~b}$ ), we examined its binding ability to the region by a luciferase reporter assay. After transfection with pre-miR-17, the luciferase activity of the TCF3-wild-type (WT) 3'-UTR reporter was suppressed by $32 \%$, whereas antimiR-17 enhanced the activity by nearly 1.43 -fold. When the binding site was mutated, no effect was observed compared with control vector (Figure 3c). Accordingly, these data demonstrated that TCF3 is a direct target gene of miR-17.

Overexpression of TCF3 attenuates the effect of pre-miR-17 on inhibiting osteogenic differentiation in PDLSCs. To further establish a functional link between TCF3 and miR-17 in the regulation of osteogenic 

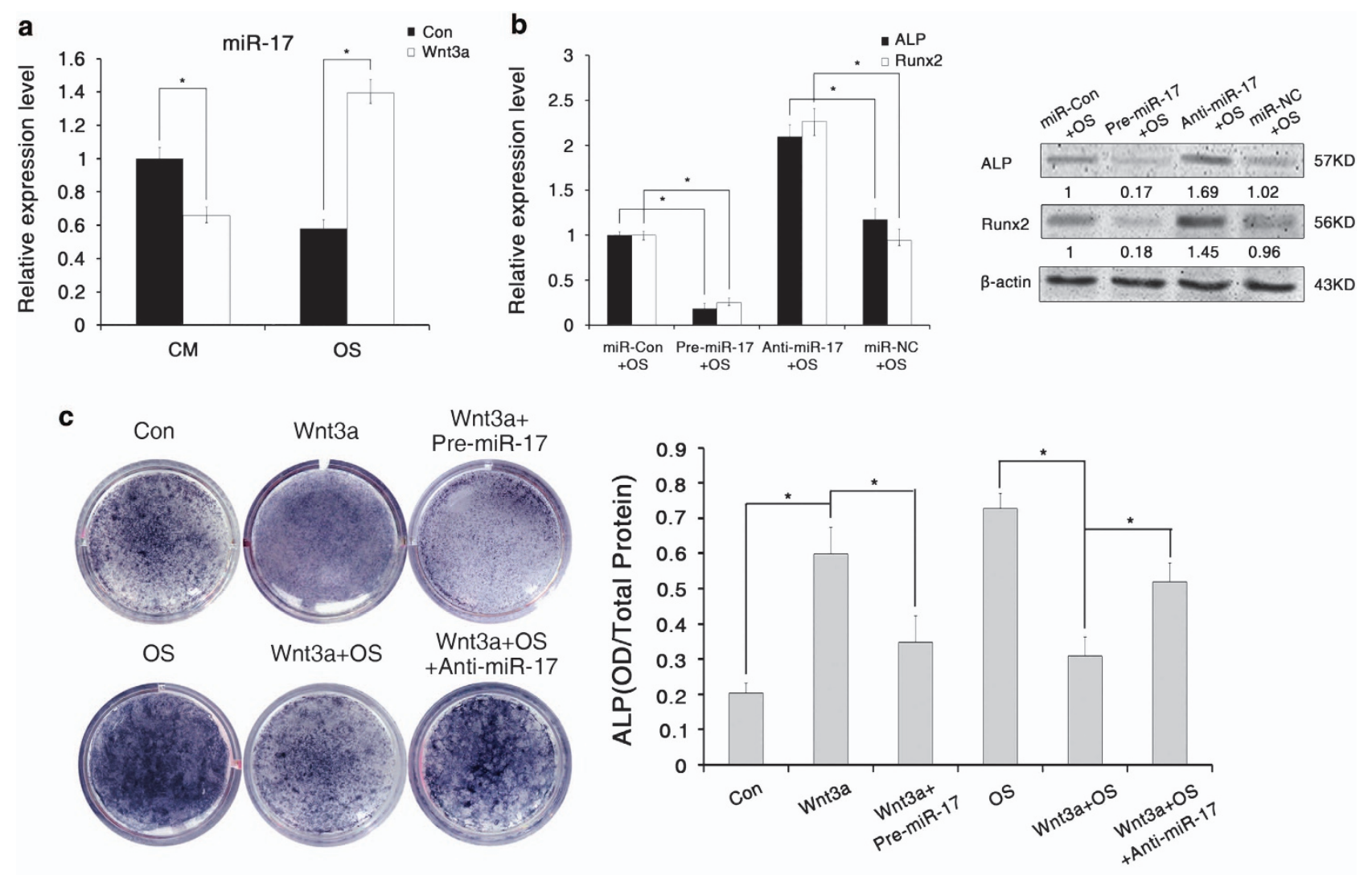

d
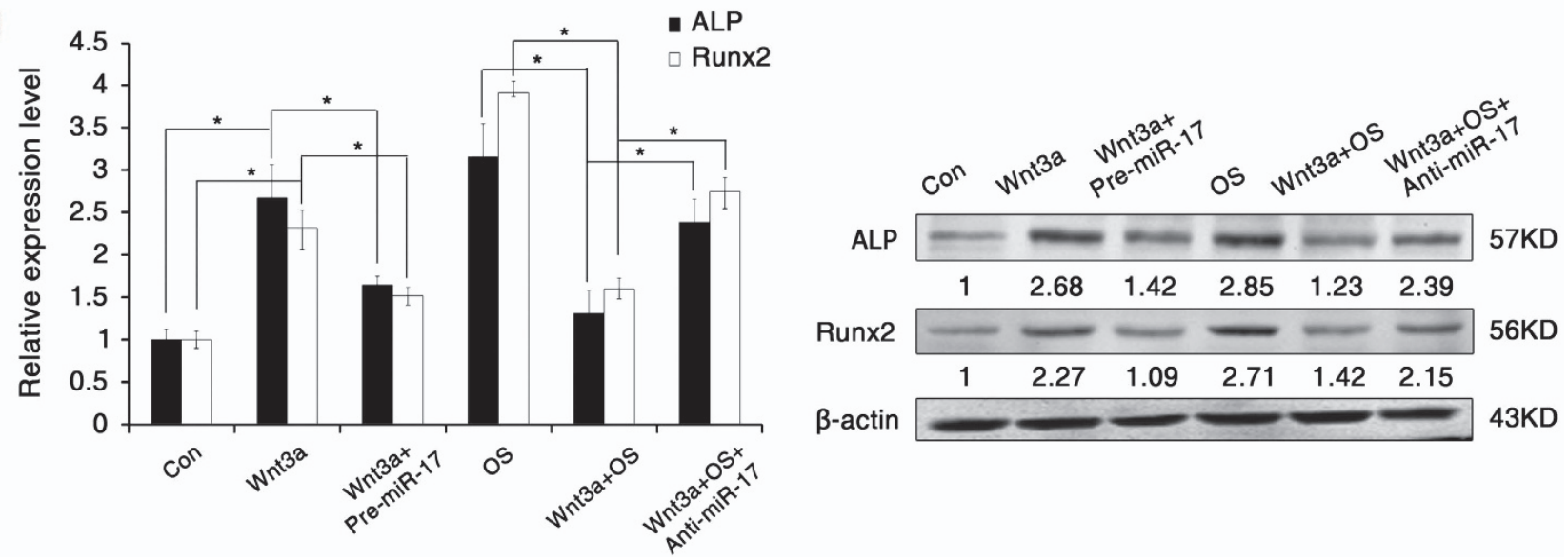

Figure 2 miR-17 could partially attenuate the effect of canonical Wnt signaling. (a) The expression level of miR-17 was measured by real-time PCR after the PDLSCs were cultured in the fully culture medium or osteogenic differentiation medium with $50 \mathrm{ng} / \mathrm{ml}$ Wnt3a for 7 days. (b) PDLSCs were transfected with pre-miR-17, anti-miR-17 and negative controls for 1 day, and then cultured in the osteogenic differentiation medium for an additional 7 days. The expression of ALP and Runx2 was measured by real-time PCR and western blot. (c and d) PDLSCs were transfected with pre-miR-17 and anti-miR-17 for 1 day and then cultured in the fully culture medium or osteogenic differentiation medium with $50 \mathrm{ng} / \mathrm{ml}$ Wnt3a for 7 days. ALP staining and activity analyses were performed at day 7 (c) and the expression of ALP and Runx2 was measured by real-time PCR and western blot (d). $\beta$-Actin and U6 were used as control for the PCR data. $\beta$-Actin was also used as a loading control. The data are shown as mean \pm S.D. ${ }^{*} P<0.05, n=3$. Con, control; CM, fully culture medium; miR-Con, siPORT reagent alone; miR-NC, miRNA negative control; OD, optical density; OS, osteogenic induction; Wnt3a, $50 \mathrm{ng} / \mathrm{ml}$ recombinant human Wnt3a

differentiation, we transfected pre-miR-17 into PDLSCs, which was stably overexpressed TCF3 first. The lentiviral vector (plenti-TCF3 (lentiviral construct for up-regulation of TCF3)) still increased the expression of TCF3 nearly fourfold after 14 days of transduction (Supplementary Figure S4a). Then, we cultured the cells in the osteogenic differentiation medium for an additional 7 or 14 days. ALP and alizarin red staining, ALP activity and calcium level analyses showed that pre-miR-17 could not effectively inhibit osteogenic differentiation when TCF3 was overexpressed (Figures 4a and b). Analyses of ALP and Runx2 expression levels confirmed this observation (Figure 4c), suggesting that the effect of pre-miR-17 on the inhibition of osteogenic differentiation was partially rescued by overexpressing 
TCF3. Taken together, our results suggest that miR-17 has its function in the regulation process primarily by acting on TCF3.

Table 1 Putative targets of miR-17

\begin{tabular}{lccc}
\hline \multicolumn{4}{c}{ Osteogenic-related pathway } \\
\cline { 2 - 4 } Gene & $\begin{array}{c}\text { Canonical Wnt } \\
\text { pathway }\end{array}$ & $\begin{array}{c}\text { BMP } \\
\text { pathway }\end{array}$ & $\begin{array}{c}\text { TGF- } \beta \\
\text { pathway }\end{array}$ \\
\hline LEF1 & + & - & - \\
TCF3 & + & - & - \\
TCF4 & + & - & - \\
\hline
\end{tabular}

Targets are predicted using TargetScan 5.0, DIANA miRGen and DIANA LAB-microTv 3.0
TCF3 plays a crucial role in miR-17 and modulates the different effect of canonical Wnt signaling on osteogenesis. Being different from the expression pattern of Smurf1, Wnt3a enhanced the expression level of TCF3 nearly 2.3-fold in the culture microenvironment, while it decreased it twofold in the differentiation microenvironment (Figure 5a). As TCF3 had close relationship with the function of miR-17 (Figure 4), we hypothesized that TCF3 may act as a reactor in miR-17 modulating the canonical Wnt pathway process. To confirm this hypothesis, we increased the expression of TCF3 stably. Then, PDLSCs were transfected with pre-miR-17 in the Wnt3a-conditioned medium for 3 days. ALP staining and activity assay showed that premiR-17 could partially attenuate the effect of Wnt3a on promoting the osteogenic differentiation in the culture
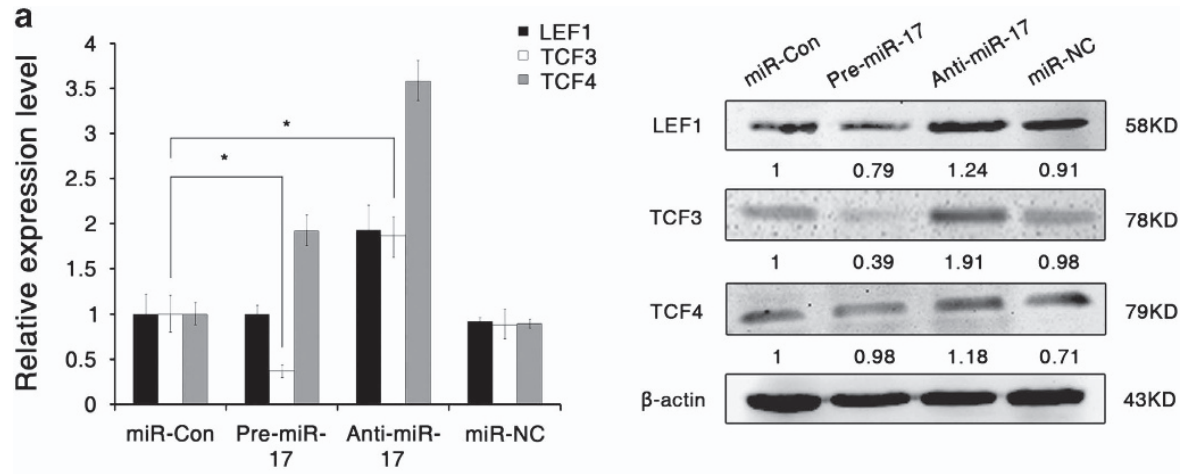

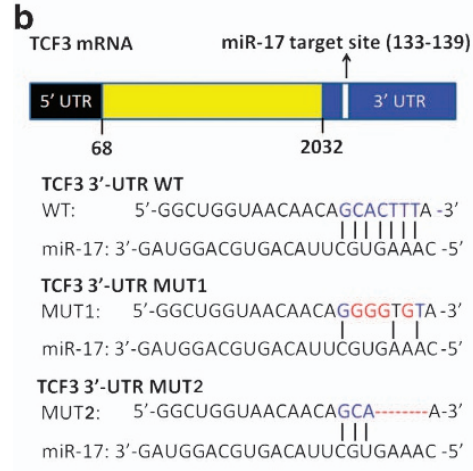

d
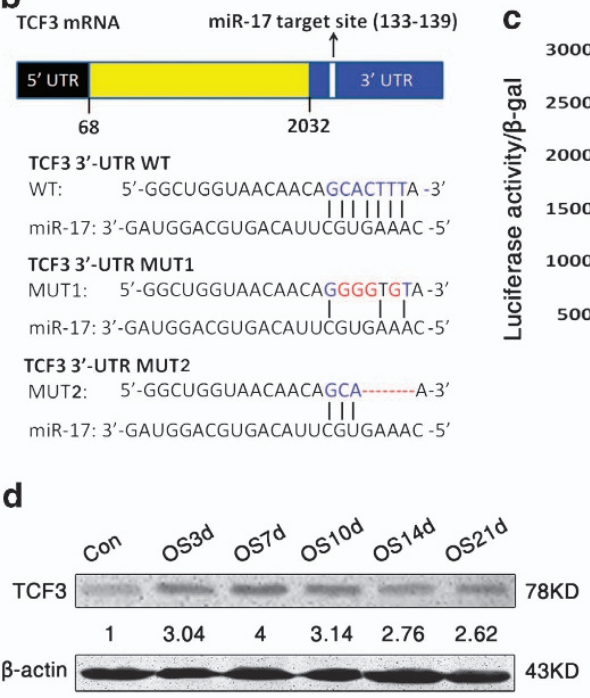

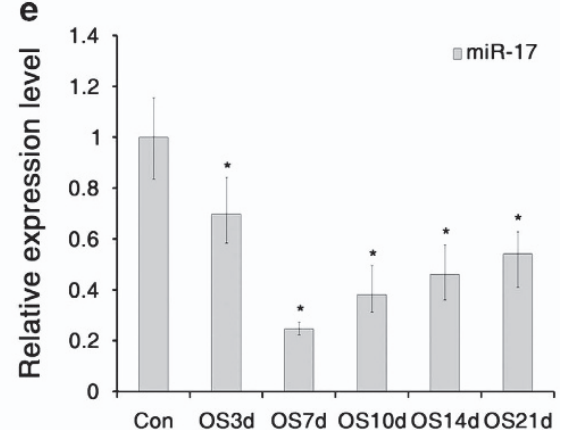

Figure 3 TCF3 was a direct target of miR-17. (a) The PDLSCs, transfected with pre-miR-17, anti-miR-17 or negative control, were harvested after $24 \mathrm{~h}$ for real-time PCR or after $48 \mathrm{~h}$ for western blot. (b) A schematic of the miR-17 putative target sites in the human TCF3 3'-UTR and the alignment of miR-17 with the WT, MUT1 and MUT2 $3^{\prime}$-UTR regions of TCF3. Two mutated nucleotides were shown in red. (c) The pMIR vector control, pMIR-TCF3-WT, pMIR-TCF3-MUT1 and pMIR-TCF3-MUT2 luciferase constructs were cotransfected with pre-miR-17, anti-miR-17 or negative control. Luciferase activity was measured after $48 \mathrm{~h}$ of transfection and normalized with $\beta$-galactosidase activity. (d) The expression pattern of TCF3 during osteogenic differentiation at the indicated time points was measured by western blot. (e) The endogenous expression of miR-17 was determined by real-time PCR at the same time points during osteogenic differentiation. The expression levels of mRNA and protein were normalized to $\beta$-actin. The data are shown as mean \pm S.D. ${ }^{*} P<0.05, n=3$. Con, control; miR-Con, siPORT reagent alone; miR-NC, miRNA negative control; OS, osteogenic differentiation; Vector, pMIR vector; WT, wild type; MUT, mutated type 

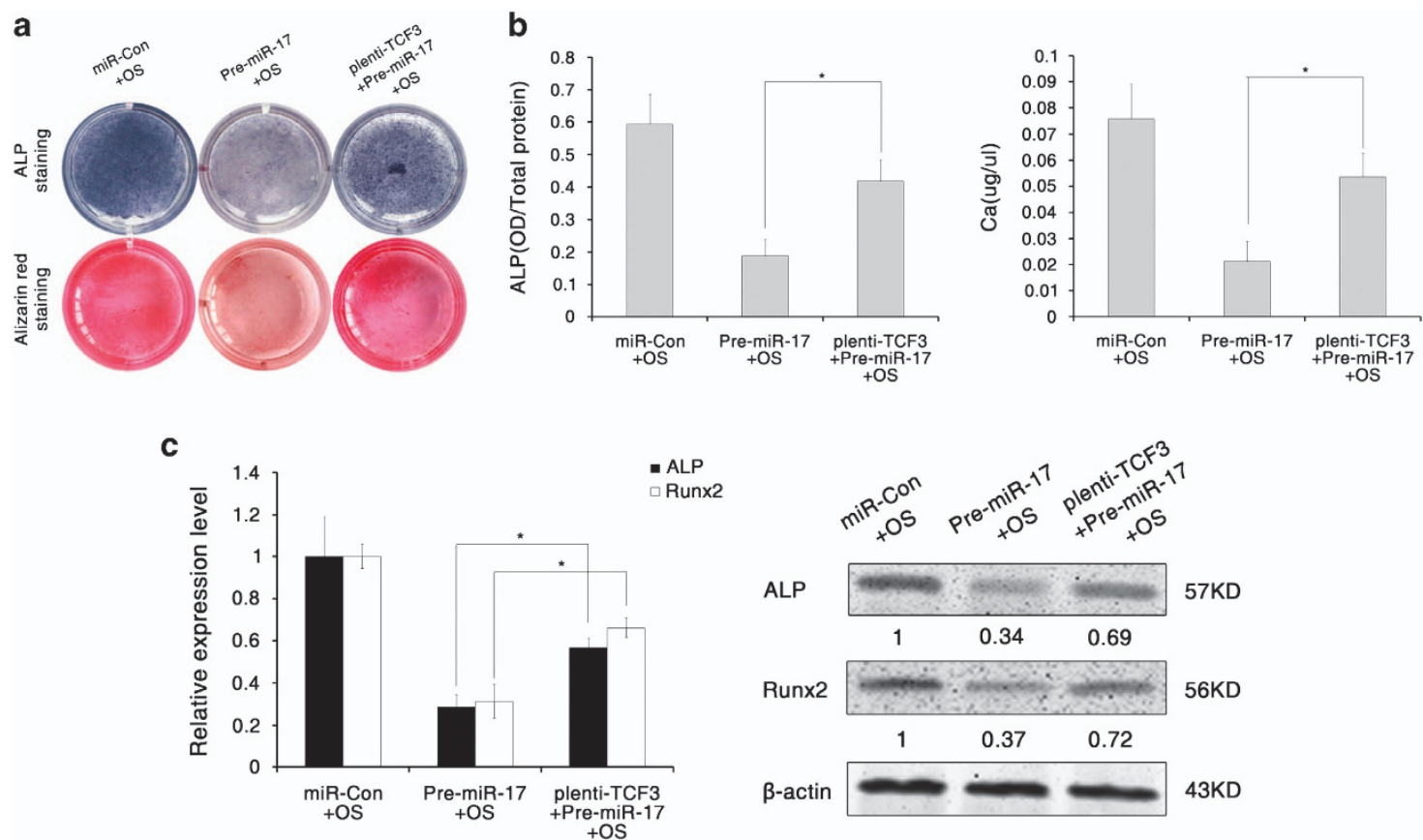

Figure 4 Overexpression of TCF3 attenuated the effect of pre-miR-17 on inhibiting osteogenic differentiation. PDLSCs were transfected with pre-miR-17 after stably overexpressing TCF3 by lentiviral construct and then cultured in osteoblastic differentiation medium for an additional 7 or 14 days. (a) Osteoblastic differentiation was determined by ALP staining at day 7 and alizarin red staining at day 14. (b) ALP activity and calcium level analyses were performed at days 7 and 14 . (c) The expressions of ALP and Runx2 were measured by real-time PCR and western blot at day 7. The expression levels of mRNA and protein were normalized to $\beta$-actin. The data are shown as mean \pm S.D. ${ }^{*} P<0.05, n=3$. miR-Con, siPORT reagent alone; OD, optical density; OS, osteoblastic induction; plenti-TCF3, lentiviral construct for upregulating TCF3

microenvironment. This based on the fact that increased level of TCF3 via Wnt3a was decreased by pre-miR-17. However, after upregulation of TCF3, pre-miR-17 did not attenuate the effect of Wnt3a on promoting osteogenic differentiation effectively (Figure 5b). PCR and western blot also supported these results (Figure $5 \mathrm{c}$ ), which suggested that miR-17 reversed the effect of Wnt3a on osteogenic differentiation mainly through directly targeting TCF3.

TCF3 acts as a positive regulator of osteogenesis both in vitro and in vivo. The role of TCF3 in miR-17 modulating the different effect of canonical Wnt signaling (Figure 5) and the increased level of TCF3 during osteogenic differentiation (Figure 3d) prompted us to investigate its function in osteogenesis. We constructed another lentiviral vector (sh-TCF3 (lentiviral construct for down-regulation of TCF3)) to induce a stable knockdown of TCF3 in PDLSCs (Supplementary Materials). The lentiviral construct (sh-TCF3-1076) decreased the TCF3 expression level 5.5-fold compared with the control (Supplementary Figure S4b). Then, we cultured the cells in osteogenic differentiation medium for an additional 7 or 14 days. ALP and alizarin red staining, ALP activity, calcium level analyses and the expression of ALP and Runx2 showed that the osteogenic differentiation of PDLSCs was suppressed after transducing sh-TCF3 in vitro (Figures 6a-c).

Next, we expended this study for ectopic bone formation in vivo. The PDLSCs transduced with sh-TCF3 or control vector were loaded onto ceramic bovine bone (CBB) powder scaffolds and implanted in NOD/SCID mice for 8 weeks.
The control group was found to form a considerable amount of bone tissue around the CBB powder. The new bone tissue was stained red using hematoxylin and eosin (H\&E) staining and appeared blue with Masson's Trichrome staining, but upon maturation, it would appear red (Figure 6d). The sh-TCF3-transduced PDLSCs formed a small number of thread-like collagen fibers that could not form any bone-like structure around the surface of the CBB powders. Because these fibers also contained collagen, they appeared red by H\&E staining or blue by Masson's Trichrome staining. Osteoid formation was decreased $46 \%$ in the implants treated with the sh-TCF3 compared with those treated with the vector control (Figure 6d). These data provided plenty of evidence that TCF3 promoted osteogenesis both in vitro and in vivo.

\section{Discussion}

For the first time, our data suggested that the contrary effect of canonical Wnt signaling in different microenvironments was caused by the different expression levels of miR-17. To elucidate the further functional link between miR-17 and canonical Wnt signaling, we found that TCF3, a key transcription factor of canonical Wnt pathway, was the direct target gene of miR-17. Furthermore, overexpression of TCF3 partially attenuated the effect of pre-miR-17 on modulating canonical Wnt signaling. Finally, we demonstrated that TCF3 promoted osteogenesis like the other members in TCF family of canonical Wnt pathway. ${ }^{13,14}$ In brief, TCF3 was the crucial node of miR-17-canonial Wnt signaling regulation loop (Figure 7). 

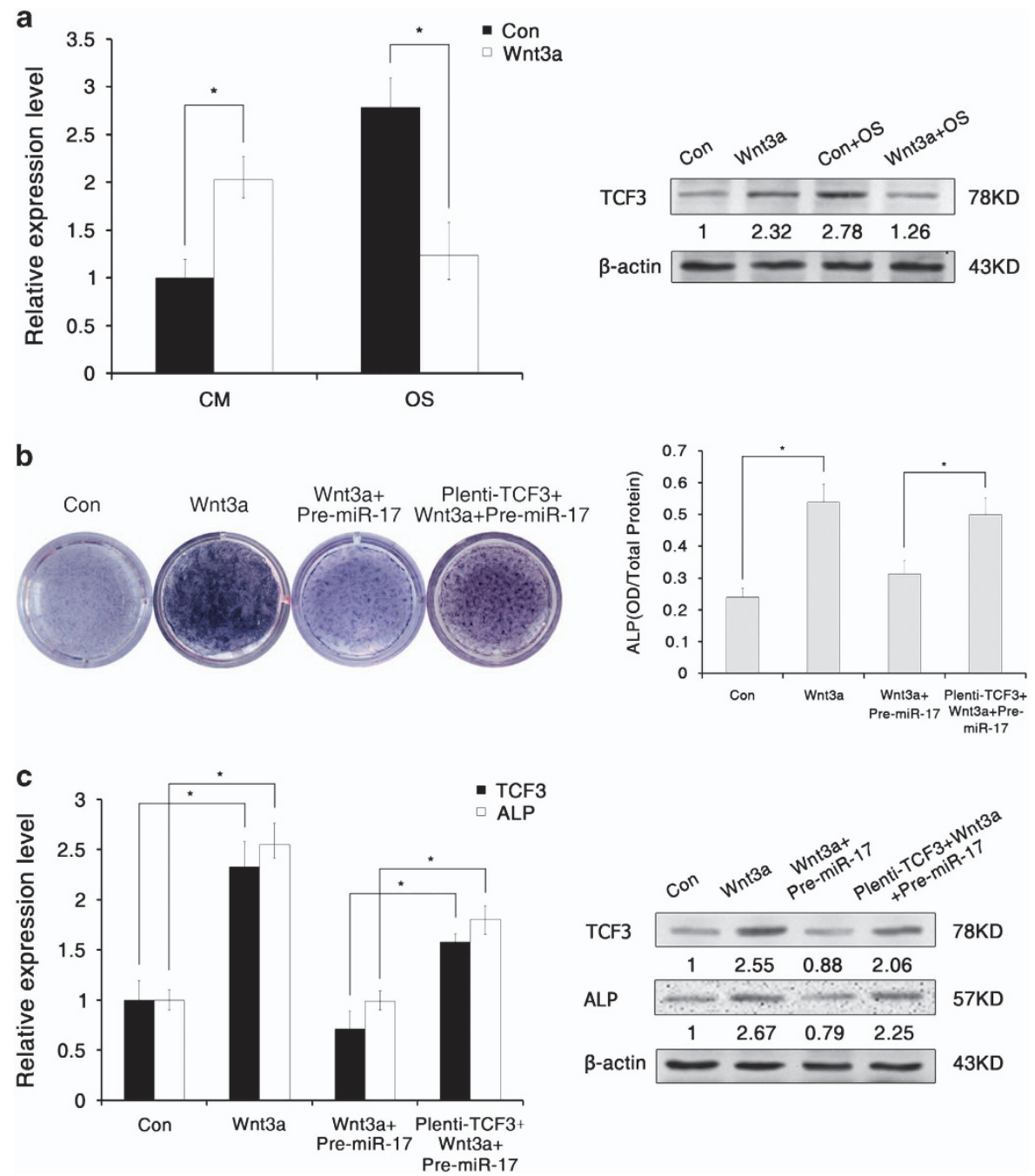

Figure 5 miR-17 modulated the contrary effect of canonical Wnt signaling mainly through targeting TCF3. (a) The expression level of TCF3 was measured by real-time PCR and western blot after the PDLSCs were cultured in the fully culture medium or osteogenic differentiation medium with $50 \mathrm{ng} / \mathrm{ml} \mathrm{Wnt3a} \mathrm{for} 7$ days. (b and c) PDLSCs were transfected with pre-miR-17 after stably overexpressing TCF3 by lentiviral construct and then cultured in the fully culture medium with $50 \mathrm{ng} / \mathrm{ml}$ Wnt3a for an additional 7 days. ALP staining and activity analyses were performed at day 7 (b) and the expression of TCF3 and ALP was measured by real-time PCR and western blot (c). The expression levels of mRNA and protein were normalized to $\beta$-actin. The data are shown as mean \pm S.D. ${ }^{*} P<0.05, n=3$. CM, fully culture medium; OD, optical density; OS, osteogenic induction; plenti-TCF3, lentiviral construct for upregulating TCF3; Wnt3a, $50 \mathrm{ng} / \mathrm{ml}$ recombinant human Wnt3a

For a long time, the in vitro effects of canonical Wnt pathway on osteogenic differentiation of MSCs are controversial. Both stimulation $^{23}$ and inhibition ${ }^{24}$ of it have been observed depending on the inducing time and differentiation stage of the cells. Furthermore, Liu et al. ${ }^{8}$ demonstrated that low-dose Wnt3a promoted osteogenic differentiation, while high dose Wnt3a inhibited it, ${ }^{8}$ suggesting that the canonical Wnt pathway regulated differentiation of human MSCs also in a dosedependent manner. Our research team previously found that the different culture environment led to the opposite effect of canonical Wnt signaling on promoting or inhibiting osteogenic differentiation of PDLSCs. ${ }^{9}$ The result was consistent with this study. PDLSCs are an MSC population derived from the periodontal tissue. ${ }^{25}$ They possess all MSC characteristics. However, PDLSCs are a little different from bone marrow MSCs in terms of osteogenesis and immunomodulatory properties, ${ }^{26-28}$ as they are derived from different tissue types. We found that the microenvironment was prone to influence the characteristics of PDLSCs than bone marrow MSCs (unpublished data). On the basis of these results, we put forward the idea that different microenvironments lead to different effects of canonical Wnt signaling, and the mechanism of that was the key point of our further research.

The microenvironment plays a critical role in the regulation of cell fate and subsequent tissue formation, ${ }^{29-31}$ and various signaling molecules were ignited subsequently. Nemeth et $a .^{32}$ suggested that modulation of Wnt signaling could affect the microenvironment, which led to the changes of stem cells. ${ }^{32}$ In addition, Edwards et al. ${ }^{33}$ raised the possibility that response of tumor cells to the Wnt signaling was highly dependent on the microenvironment and the local balance of agonists and antagonists of this pathway. ${ }^{33}$ However, the 
a

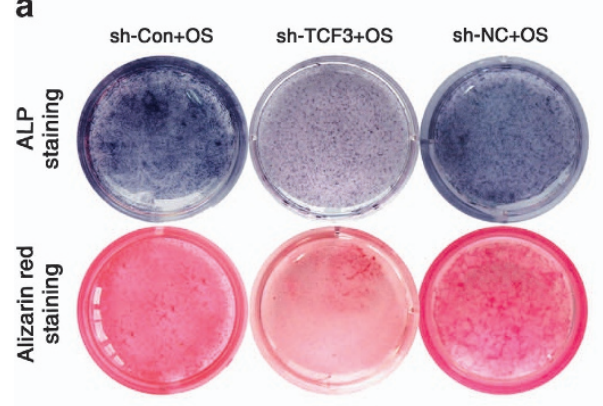

b

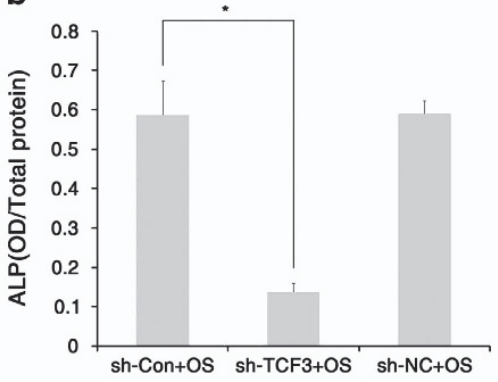

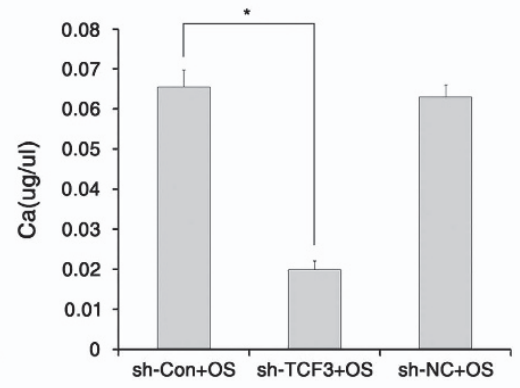

c
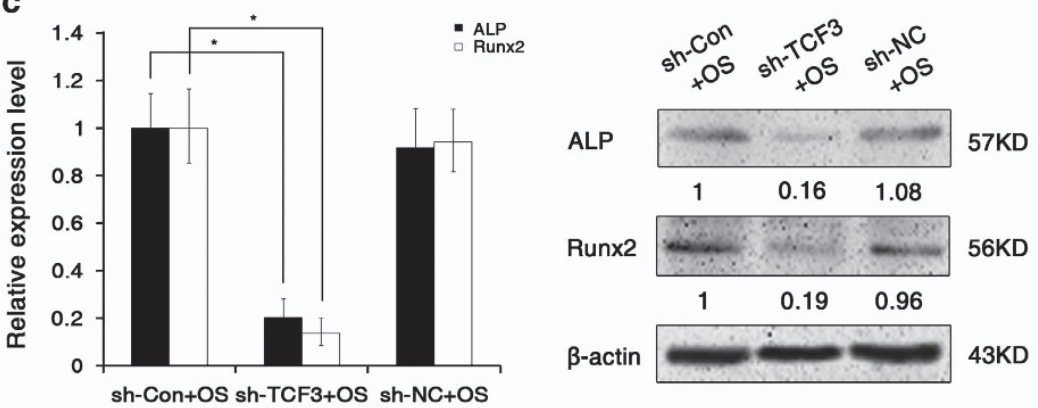

a
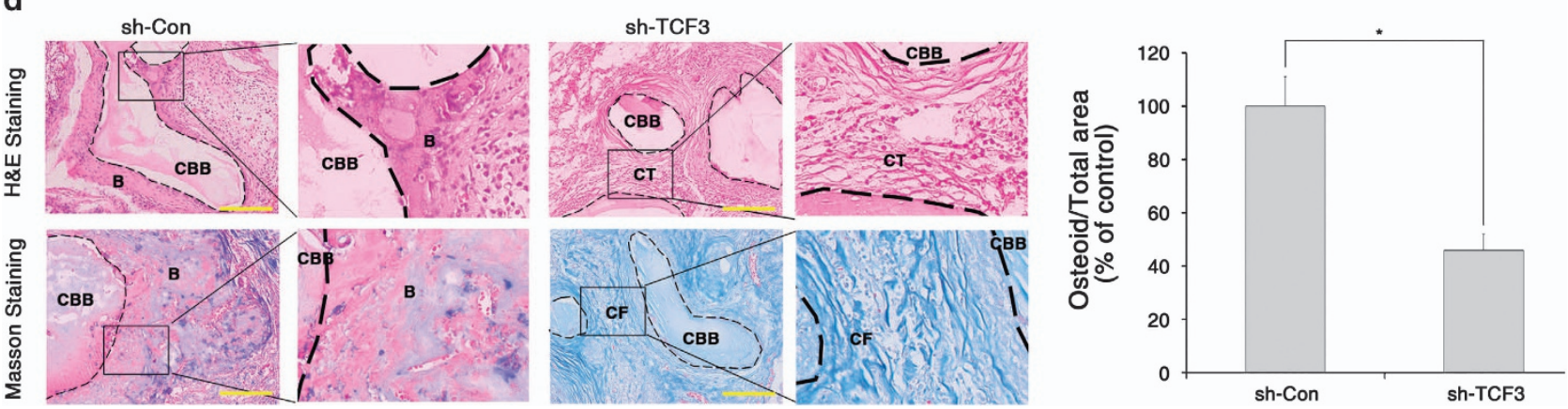

Figure 6 TCF3 promoted osteogenic differentiation of PDLSCs in vitro and ectopic bone formation in vivo. To confirm the effect of TCF3 on osteogenesis, PDLSCs were transduced with lentiviral constructs for 3 days and then cultured in osteogenic differentiation medium for an additional 7 or 14 days. (a) Osteogenic differentiation was determined by ALP staining at day 7 and alizarin red staining at day 14. (b) ALP activity and calcium level analyses were performed at days 7 and 14 . (c) The expressions of ALP and Runx2 were confirmed by real-time PCR and western blot analyses at day 7 . The expression levels of mRNA and protein were normalized to $\beta$-actin. ${ }^{*} P<0.05$, $n=3$. (d) PDLSCs were transduced with sh-Con or sh-TCF3 for 3 days and then implanted into non-obese diabetic/severe-combined immunodeficient (NOD/SCID) mice. H\&E and Masson's Trichrome staining was performed after 8 weeks of implantation. Osteoid formation was quantified as total osteoid volume per total volume by Masson's staining. Scale bar: $50 \mu \mathrm{m}$. Six implants per treatment were engrafted into the mice, and three sections of each implant were quantified to minimize variations within the implants. Data are shown as the means \pm S.D. ${ }^{*} P<0.05, n=6$. OD, optical density; OS, osteogenic induction; sh-Con, virus produced from the two packaging vectors; sh-NC, lentivirus negative control; sh-TCF3, lentiviral construct for downregulating TCF3

relationship between Wnt signaling and the microenvironment is more similar to a bidirectional network rather than a one way cascade. In our study, we observed that the microenvironment in turn regulated the effect of canonical Wnt signaling. As we know, miRNAs more frequently target signaling proteins than other common genes. ${ }^{34,35}$ In addition, we found that the role of miR-17 in simulation or inhibition of osteoblast differentiation was determined by the microenvironment described previously. ${ }^{22}$ In this study, we observed that Wnt3a decreased the expression of miR-17 in the fully culture medium, whereas increased its level in osteogenic differentiation medium. It suggested that miR-17 had close relationship with Wnt signaling. Furthermore, the contrary effect of Wnt signaling on osteogenesis could be attenuated by pre-miR-17 or anti-miR-17. In other words, miR-17 could reverse the effect of 'on/off' signals in the Wnt pathway. As the miRNA processing was faster than the signaling pathway cascade activation, miRNAs were affected with a shorter delay than transcriptional pathways and were more sensitive to the microenvironment. ${ }^{36}$ We conferred that miR-17 acted as a 'toggle switch' of paradoxical effect of canonical Wnt signaling in different microenvironments.

miRNAs negatively regulated the expression of its target genes to play important roles in a wide array of biological processes. ${ }^{37,38}$ Smurf1 was confirmed as a target gene of miR-17 in our previous research. ${ }^{22}$ However, it was not affected by Wnt signaling in this study. Fortunately, we validated TCF3, which belonged to the TCF/LEF family of 
$\square$ Up regulation

Down regulation

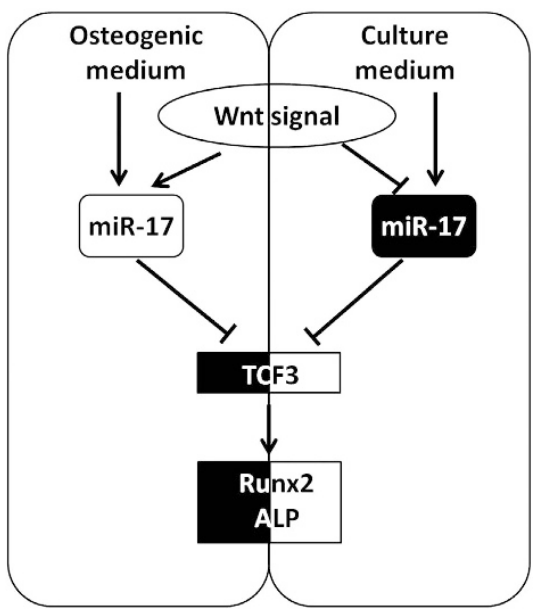

Figure 7 Working model of miR-17 in the modulating contrary effect of canonical Wnt signaling in different microenvironments

canonical Wnt pathway, as another direct target of miR-17 in PDLSCs. Recently, TCF3 has been proven to be vital for development. ${ }^{39}$ In mouse, TCF3-deficient embryos undergo gastrulation but exhibit variable degrees of defects. ${ }^{40}$ While TCF3 knockdown-derived mouse ESC self-renewal was enhanced, its cell differentiation was inhibited. ${ }^{41,42}$ In humans, the expression of TCF3 has been associated with poorly differentiated mammary gland tumors. ${ }^{43}$ As transcription factors, TCF/LEF family combined with $\beta$-catenin to regulate downstream target genes. Solberg et al. ${ }^{39}$ proved that there existed a direct physical interaction between $\beta$-catenin and TCF3 in the nucleus in mouse neural stem cells and human HEK 293 cells. As TCF3 possesses a biochemical activity not shared by other TCF/LEF members, ${ }^{44}$ it could inhibit the rate of $\beta$-catenin degradation via its competition with axin and adenomatous polyposis for $\beta$-catenin binding. ${ }^{45}$ These data elucidated that TCF3 interacted with canonical Wnt pathway directly. Usually, the expression level of TCF/LEF family was upregulated after activation of the canonical Wnt pathway. Interestingly, our data showed that Wnt3a could decrease TCF3 expression level twofold in the differentiation microenvironment. As TCF3 was a direct target gene of miR-17, we conferred that the downregulation of TCF3 may be caused by the increased level of miR-17 after adding Wnt3a. However, we remain fully aware that miR-17 or TCF3 may not be the only downstream target genes of Wnt pathway, and our result does not exclude the possibility that other target genes, such as other TCF/LEF members yet to be identified, may additionally account for the discrepancy in the result.

Our results demonstrated that miR-17 modulated the osteogenesis of PDLSCs mainly through directly targeting TCF3. Besides, we observed that pre-miR-17 did not attenuate the effect of Wnt3a on promoting osteogenic differentiation of PDLSCs effectively after upregulation of TCF3. In conclusion, we conferred that TCF3 was a key point of miR-17 modulating the contrary effect of canonical Wnt signaling in different microenvironments. Importantly, we elucidated that TCF3 acted as a positive regulator of osteogenesis in PDLSCs for the first time. The result was similar to TCF1 and TCF4, which promoted MSCs' osteogenesis by inducing endogenous Runx2 expression before. $^{13-15,46}$ Nevertheless, the additional relationship between the microenvironment and miR-17 should be investigated in future studies, maybe focus on the epigenetics.

Totally, canonical Wnt signal acts as a positive or negative regulator of PDLSCs' osteogenesis in different microenvironments depending on the expression level of miR-17. That is because miRNAs were more sensitive and rapidly respond to the microenvironment than the signaling complex. Previously, we confirmed that the function of miR-17 changed depending on the microenvironment. ${ }^{22}$ Combined with this study, we deduced that miR-17 acted as a 'toggle switch' in modulating signaling pathways. This understanding may have applications in miRNAs regulating so far enigmatic, and necessarily holistic, aspects of biological process, such as differentiation, homeostasis and regeneration. Furthermore, our results may have a marked impact on miRNA-based therapy for bonerelated disorders.

\section{Materials and Methods}

Cell culture and identification. See Supplementary Methods for more details.

Mineralization assay. See Supplementary Methods for more details.

Transfection assay. The following precursor and inhibitor miRNAs were used: pre-miR-17, pre-miR negative control, anti-miR-17 and anti-miR negative control (Ambion, Austin, TX, USA). Pre-miR-17 and anti-miR-17 were transfected into PDLSCs at $50 \mathrm{nM}$ concentration with the siPORT NeoFX Transfection Agent (Ambion). The medium was replaced $8 \mathrm{~h}$ later and the cells were harvested for mRNA analysis after $24 \mathrm{~h}$ transfection and for protein analysis after $48 \mathrm{~h}$.

Lentiviral vector construction and transduction. To construct a lentiviral vector for human TCF3, TCF3 was amplified by PCR from human genomic DNA (Supplementary Methods). The PCR product was digested with the restriction enzymes Agel and EcoRl and inserted into the PLKO.1 vector (Invitrogen, Carlsbad, CA, USA) and was also digested with Kpnl and EcoRV and inserted into the plenti 6.3/v5-DEST vector (Invitrogen). The inserted fragments were verified by Sanger sequencing. A lentiviral construct containing the scrambled TCF3 sequence was used as a negative control. The lentivirus was produced by cotransfecting 293T cells with the transfer vector and two packaging vectors (i.e., psPAX2 and pMD2.G). The virus was subsequently purified by ultracentrifugation. PDLSCs were plated in 6-well plates and transduced with lentiviral constructs and $5 \mu \mathrm{g} / \mathrm{ml}$ polybrene (Sigma, St. Louis, MO, USA).

Real-time PCR analysis. See Supplementary Methods for details.

Western blot analysis. See Supplementary Methods for details.

Luciferase assay. For the luciferase reporter assays, PCR fragments, including the TCF3 $3^{\prime}$-UTR containing the putative binding site for miR-17 with WT or mutant (MUT) sequences, were cloned into the pMIR-Report luciferase plasmid (Ambion). The following oligonucleotides were used: TCF3-WT (5'-GGC UGGUAACAACAGCACTTTA-3'), TCF3-MUT1 (5'-GGCUGGUAACAACAGGGG TGTA-3') and TCF3-MUT2 (5' -GGCUGGUAACAACAGCA $\times \times \times \times$ A-3', where $\times$ means deleted nucleotides). PDLSCs were cotransfected with the luciferase vector, the pMIR-Report $\beta$-gal control plasmid (as a normalization control) (Ambion) and the pre-miR-17 and anti-miR-17 using Lipofectamine 2000 (Invitrogen). Cells were transfected in triplicates, and each transfected well was 
assayed in triplicates after $24 \mathrm{~h}$ using the Bright-Glo Luciferase Assay System (Promega, Madison, WI, USA). Luciferase activities were measured in a Microplate Luminometer (Promega).

In vivo bone formation assay. All of the procedures that involved animals were approved by the Animal Use and Care Committee of the Fourth Military Medical University (license number: SCXK 2007-007). For a single transplant complex, the PDLSCs were transduced with sh-TCF3 lentiviral construct as described above, and then cultured for 3 days. Approximately $5.0 \times 10^{6}$ cells were mixed with $40 \mathrm{mg}$ of CBB powders (Research and Development Center for Tissue Engineering, Fourth Military Medical University, Xi'an, China) and implanted into subcutaneous pockets on the backs of the 8-week NOD/SCID mice (Fourth Military Medical University). ${ }^{47}$ As a control, PDLSCs from the same sources that had been treated with the lentivirus control were implanted into the other side of the same host. The implants were taken out 8 weeks after transplantation, fixed with $4 \%$ paraformaldehyde and decalcified with buffered $10 \%$ EDTA (pH 6.0). For the histological analyses, the sections were stained with H\&E or Masson's Trichrome staining (BaSO Diagnostic Inc., Guangdong, China) according to the manufacturer's instructions.

Statistics. The data are presented as the mean \pm S.D. Comparisons were made using a $t$-test or one-way ANOVA for experiments with more than three groups. All of the experiments were repeated three times, and representative experiments are shown. The differences were considered to be significant when $P<0.05$. The $P$-values were adjusted using the Bonferroni method.

\section{Conflict of Interest}

The authors declare no conflict of interest.

Acknowledgements. This work was supported by grants from the National Basic Research Program (973 Program) (2011CB964700 and 2010CB944802), the Nature Science Foundation of China (81020108019, 31030033 and 31200972) and a grant from the National Institute of Dental and Craniofacial Research, National Institutes of Health and Department of Health and Human Services (R01DE019932 to SS).

1. Jensen ED, Gopalakrishnan R, Westendorf JJ. Regulation of gene expression in osteoblasts. Biofactors 2010; 36: 25-32.

2. Soltanoff CS, Yang S, Chen W, Li YP. Signaling networks that control the lineage commitment and differentiation of bone cells. Crit Rev Eukaryot Gene Expr2009; 19: 1-46.

3. Krishnan V, Bryant HU, Macdougald OA. Regulation of bone mass by Wnt signaling. J Clin Invest 2006; 116: 1202-1209.

4. Yang F, Yang D, Tu J, Zheng Q, Cai L, Wang L. Strontium enhances osteogenic differentiation of mesenchymal stem cells and in vivo bone formation by activating Wnt/catenin signaling. Stem Cells 2011; 29: 981-991.

5. Bennett CN, Ouyang $H$, Ma YL, Zeng Q, Gerin I, Sousa KM et al. Wnt10b increases postnatal bone formation by enhancing osteoblast differentiation. J Bone Miner Res 2007; 22: 1924-1932.

6. Guo AJ, Choi RC, Cheung AW, Chen VP, Xu SL, Dong TT et al. Baicalin, a flavone, induces the differentiation of cultured osteoblasts: an action via the Wnt/beta-catenin signaling pathway. J Biol Chem 2011; 286: 27882-27893.

7. de Boer J, Siddappa R, Gaspar C, van Apeldoorn A, Fodde R, van Blitterswijk C. Wnt signaling inhibits osteogenic differentiation of human mesenchymal stem cells. Bone 2004; 34: 818-826.

8. Liu G, Vijayakumar S, Grumolato L, Arroyave R, Qiao H, Akiri G et al. Canonical Wnts function as potent regulators of osteogenesis by human mesenchymal stem cells. J Cell Biol 2009; 185: 67-75.

9. Liu N, Shi S, Deng M, Tang L, Zhang G, Liu N et al. High levels of $\beta$-catenin signaling suppress osteogenic differentiation of periodontal ligament stem cells in inflammatory microenvironments through inhibition of the noncanonical Wnt pathway. J Bone Miner Res 2011; 26: 2082-2095.

10. Ling L, Nurcombe V, Cool SM. Wnt signaling controls the fate of mesenchymal stem cells. Gene 2009; 433: 1-7.

11. Moon RT, Kohn AD, De Ferrari GV, Kaykas A. WNT and beta-catenin signalling: diseases and therapies. Nat Rev Genet 2004; 5: 691-701.

12. Jacques BE, Puligilla C, Weichert RM, Ferrer-Vaquer A, Hadjantonakis AK, Kelley MW et al. A dual function for canonical Wnt/ $\beta$-catenin signaling in the developing mammalian cochlea. Development 2012; 139: 4395-4404.
13. Gaur T, Lengner CJ, Hovhannisyan H, Bhat RA, Bodine PV, Komm BS et al. Canonical WNT signaling promotes osteogenesis by directly stimulating Runx2 gene expression. J Biol Chem 2005; 280: 33132-33140.

14. McCarthy TL, Centrella M. Novel links among Wnt and TGF-beta signaling and Runx2. Mol Endocrinol 2010; 24: 587-597.

15. Kahler RA, Westendorf JJ. Lymphoid enhancer factor-1 and beta-catenin inhibit Runx2dependent transcriptional activation of the osteocalcin promoter. J Biol Chem 2003; 278: 11937-11944.

16. Li Z, Hassan MQ, Volinia S, van Wijnen AJ, Stein JL, Croce CM et al. A microRNA signature for a BMP2-induced osteoblast lineage commitment program. Proc Natl Acad Sci USA 2008; 105: 13906-13911.

17. Du T, Zamore PD. Beginning to understand microRNA function. Cell Res 2007; 17: $661-663$.

18. Neilson JR, Sharp PA. Small RNA regulators of gene expression. Cell 2008; 134: 899-902.

19. Huang J, Zhao L, Xing L, Chen D. MicroRNA-204 regulates Runx2 protein expression and mesenchymal progenitor cell differentiation. Stem Cells 2010; 28: 357-364.

20. Tomé M, López-Romero P, Albo C, Sepúlveda JC, Fernández-Gutiérrez B, Dopazo A et al. miR-335 orchestrates cell proliferation, migration and differentiation in human mesenchymal stem cells. Cell Death Differ 2011; 18: 985-995.

21. Zhang JF, Fu WM, He ML, Xie WD, Lv Q, Wan G et al. MiRNA-20a promotes osteogenic dilerentiation of human mesenchymal stem cells by co-regulating BMP signalling. RNA Biol 2011; 8: 829-838.

22. Liu Y, Liu W, Hu C, Xue Z, Wang G, Ding B et al. MiR-17 modulates osteogenic differentiation through a coherent feed-forward loop in mesenchymal stem cells isolated from periodontal ligaments of patients with periodontitis. Stem Cells 2011; 29: 1804-1816.

23. Gregory CA, Gunn WG, Reyes E, Smolarz AJ, Munoz J, Spees JL et al. How Wnt signaling affects bone repair by mesenchymal stem cells from the bone marrow. Ann NY Acad Sci 2005; 1049: 97-106.

24. Boland GM, Perkins G, Hall DJ, Tuan RS. Wnt 3a promotes proliferation and suppresses osteogenic differentiation of adult human mesenchymal stem cells. J Cell Biochem 2004; 93: $1210-1230$.

25. Seo BM, Miura M, Gronthos S, Bartold PM, Batouli S, Brahim J et al. Investigation of multipotent postnatal stem cells from human periodontal ligament. Lancet 2004; 364 : 149-155.

26. Huang GT, Gronthos S, Shi S. Mesenchymal stem cells derived from dental tissues vs. those from other sources: their biology and role in regenerative medicine. $J$ Dent Res 2009; 88: 792-806.

27. Wada N, Menicanin D, Shi S, Bartold PM, Gronthos S. Immunomodulatory properties of human periodontal ligament stem cells. J Cell Physiol 2009; 219: 667-676.

28. Liu Y, Wang L, Kikuiri T, Akiyama K, Chen C, Xu X et al. Mesenchymal stem cell-based tissue regeneration is governed by recipient $T$ lymphocytes via IFN- $\gamma$ and TNF- $\alpha$. Nat Med 2011; 17: 1594-1601.

29. Burdick JA, Vunjak-Novakovic G. Engineered microenvironments for controlled stem cell differentiation. Tissue Eng Part A 2009; 15: 205-219.

30. Yamashita A, Nishikawa S, Rancourt DE. Microenvironment modulates osteogenic cell lineage commitment in differentiated embryonic stem cells. PLoS One 2010; 5: e9663.

31. Hu Y, Cai K, Luo Z, Zhang Y, Li L, Lai M et al. Regulation of the differentiation of mesenchymal stem cells in vitro and osteogenesis in vivo by microenvironmental modification of titanium alloy surfaces. Biomaterials 2012; 33: 3515-3528.

32. Nemeth MJ, Mak KK, Yang Y, Bodine DM. Beta-catenin expression in the bone marrow microenvironment is required for long-term maintenance of primitive hematopoietic cells. Stem Cells 2009; 27: 1109-1119.

33. Edwards CM, Edwards JR, Lwin ST, Esparza J, Oyajobi BO, McCluskey B et al. Increasing Wnt signaling in the bone marrow microenvironment inhibits the development of myeloma bone disease and reduces tumor burden in bone in vivo. Blood 2008; 111: 2833-2842.

34. Cui $Q$, Yu Z, Purisima EO, Wang E. Principles of microRNA regulation of a human cellular signaling network. Mol Syst Biol 2006; 2: 46.

35. Mendell JT, Olson EN. MicroRNAs in stress signaling and human disease. Cell 2012; 148 : 1172-1187.

36. Inui M, Martello G, Piccolo S. MicroRNA control of signal transduction. Nat Rev Mol Cell Biol 2010; 11: 252-263.

37. Liu L, Wang Y, Fan H, Zhao X, Liu D, Hu Y et al. MicroRNA-181a regulates local immune balance by inhibiting proliferation and immunosuppressive properties of mesenchymal stem cells. Stem Cells 2012; 30: 1756-1770.

38. Li Y, Vecchiarelli-Federico LM, Li YJ, Egan SE, Spaner D, Hough MR et al. The miR-17-92 cluster expands multipotent hematopoietic progenitors whereas imbalanced expression of its individual oncogenic miRNAs promotes leukemia in mice. Blood 2012; 119: 4486-4498.

39. Solberg N, Machon O, Machonova O, Krauss S. Mouse Tcf3 represses canonical Wnt signaling by either competing for $\beta$-catenin binding or through occupation of DNA-binding sites. Mol Cell Biochem 2012; 365: 53-63.

40. Andoniadou CL, Signore M, Young RM, Gaston-Massuet C, Wilson SW, Fuchs E et al. HESX1- and TCF3-mediated repression of Wnt/ $\beta$-catenin targets is required for normal development of the anterior forebrain. Development 2011; 138: 4931-4942. 
41. Yi F, Pereira L, Merrill BJ. Tcf3 functions as a steady-state limiter of transcriptional programs of mouse embryonic stem cell self-renewal. Stem Cells 2008; 26: 1951-1960.

42. Tam WL, Lim CY, Han J, Zhang J, Ang YS, Ng HH et al. T-cell factor 3 regulates embryonic stem cell pluripotency and self-renewal by the transcriptional control of multiple lineage pathways. Stem Cells 2008; 26: 2019-2031.

43. Ben-Porath I, Thomson MW, Carey VJ, Ge R, Bell GW, Regev A et al. An embryonic stem cell-like gene expression signature in poorly differentiated aggressive human tumors. Nat Genet 2008; 40: 499-507.

44. Yi F, Pereira L, Hoffman JA, Shy BR, Yuen CM, Liu DR et al. Opposing effects of Tcf3 and Tcf1 control Wnt stimulation of embryonic stem cell self-renewal. Nat Cell Biol 2011; 13 762-770.

45. Lee E, Salic A, Kirschner MW. Physiological regulation of $\beta$-catenin stability by Tcf3 and CK1epsilon. J Cell Biol 2001; 154: 983-993.
46. Reinhold Mi, Naski MC. Direct interactions of Runx2 and canonical Wnt signaling induce FGF18. J Biol Chem 2007; 282: 3653-3663.

47. Yang $\mathrm{ZH}$, Jin F, Zhang XJ, Ma DD, Han $\mathrm{C}$, Huo $\mathrm{N}$ et al. Tissue engineering of cementum/ periodontal-ligament complex using a novel three-dimensional pellet cultivation system for human periodontal ligament stem cells. Tissue Engineering Part C 2009; 15: 571-581.

(c) (i) (s) $\ominus$ Cell Death and Disease is an open-access journal BY NC No published by Nature Publishing Group. This work is licensed under a Creative Commons Attribution-NonCommercialNoDerivs 3.0 Unported License. To view a copy of this license, visit http://creativecommons.org/licenses/by-nc-nd/3.0/

Supplementary Information accompanies this paper on Cell Death and Disease website (http://www.nature.com/cddis) 